\title{
Surface-Patterning of Nanocomposite Hydrogel Film by Direct Replica Molding and Subsequent Change in Pattern Size
}

\author{
By Liyuan SoNG, ${ }^{1}$ Meifang ZHU, ${ }^{1}$ Yanmo $C H E N,{ }^{1}$ and Kazutoshi HARAGUCHI ${ }^{2, *}$
}

\begin{abstract}
Using PNIPA nanocomposite hydrogel (NC gel), which exhibits extraordinary mechanical toughness as well as high transparency and good thermal sensitivity, micrometer-scale surface-patterned NC gel films were successfully prepared by direct replica molding (direct REM) without any chemical surface treatment of the template. The surface pattern and its changes were characterized based on 3-D images and 2-D profiles generated by confocal optical microscopy. It was found that small $(\sim 1 \mu \mathrm{m})$ microstructures were precisely transferred onto the surfaces of the NC gel films without defect, and that the size of the patterns could be modified (enlarged or miniaturized) by subsequent swelling, deswelling and drying without disturbing the patterns.

KEY WORDS: Clay / Hydrogel / Nanocomposite / Patterning / Direct Replica Molding /
\end{abstract}

Polymer hydrogels are soft, wet materials which consist of a three-dimensional polymer network and a large amount of water. Among various types of polymer hydrogel, stimulussensitive hydrogels have received broad attention as functional soft materials which are useful in bio-technology, optics, sensors, analysis, micro-reactors and electoro-mechanical systems. ${ }^{1-6}$ In particular, poly $(N$-isopropylacrylamide) (PNIPA) hydrogel, which undergoes a well-defined phase transition at the lower critical solution temperature (LCST), has been widely developed as the most promising smart gel. ${ }^{7-11}$

To enhance the hydrogel's functionality, micrometer-scale surface-patterning of the hydrogel is highly desirable. ${ }^{12-14}$ So far, photolithography, which is a key micro-fabrication technology in semiconductor science, has been applied to prepare surface-patterned hydrogel by transferring a geometric pattern from a photomask, ${ }^{15-19}$ but it is only limited to photocurable monomer or polymer systems. For conventional hydrogels, an alternative technique of surface-patterning called "soft lithography" ${ }^{20,21}$ has been developed. This method relies on an elastic stamp, made of materials such as poly(dimethylsiloxane) (PDMS), and the following processes shown in Scheme 1(a): coating of a substrate with the gel precursor, stamping, and peeling off of the elastic stamp after curing the gel. Then, the microstructured hydrogel, i.e., the negative copy of the stamp pattern, is fabricated on a flat solid substrate. ${ }^{22-26}$ For instance, chemically crosslinked poly(acrylamide) gel has been molded at room temperature on a silicon plate using a PDMS template. ${ }^{24}$ Alginate and chitosan gels have been micromolded on a glass plate by release of the gelling agent from patterned agarose gel. ${ }^{25}$ Also, microstructured collagen and sugar-based gels have been fabricated on glass substrate using surface-treated PDMS stamps, through a few procedures adopted from soft lithography and modified, such as replica molding (REM), microtransfer molding ( $\mu \mathrm{TM})$ and micromolding in capillaries (MIMIC). ${ }^{26}$
On the other hand, the imprinting of microstructures on the surface of hydrogel film (separated from the solid substrate) has only succeeded in a few cases. For example, $\sim 1$-mm-thick patterned 2-hydroxyethylmethacrylate hydrogel film has been prepared using a silicon wafer stamp and the process shown in Scheme 1(b-1). ${ }^{27,28}$ Here, surface treatment of the silicon wafer with $1 \mathrm{H}, 1 \mathrm{H}, 2 \mathrm{H}, 2 \mathrm{H}$-perfluoroctyltrichlorosilane and the subsequent swelling of the hydrogel are necessary to lift the hydrogel film from the hard template, although this treatment makes it difficult to control the water content of hydrogel film. In the case of agarose gel, by the casting and molding procedure using a PDMS template shown in Schemes $1(\mathrm{~b}-2)^{29}$ and (b-3), ${ }^{30} \sim 30$-mm-thick surface-patterned agarose gel films have been prepared. However, in all the cases described above, the obtained surface-patterned hydrogel films were not mechanically tough enough for stretching or bending deformation, because of the chemically cross-linked hydrogel network. Also, the stimulus-sensitivity was not achieved in the patterned hydrogel films; i.e., stimulus-sensitive surface-patterned PNIPA hydrogel film has not been obtained.

Recently, we found that the serious disadvantages associated with chemically cross-linked PNIPA hydrogels, e.g., the mechanical fragility and low thermo-sensitivity, were dramatically improved in nanocomposite hydrogel (abbreviation: NC gel) through the in situ free radical polymerization of NIPA in the presence of exfoliated clay nanoparticles. Since NC gel consists of a unique organic (PNIPA)/inorganic (clay) network structure, in which a large number of flexible PNIPA chains are physically cross-linked via the hydrogen bonds on the clay platelets, it exhibits extraordinarily high mechanical toughness which enables it to withstand large and reversible stretching, compression and bending.

In the present study, based on PNIPA/clay nanocomposite hydrogel (NC gel) which exhibits outstanding mechanical, optical and stimulus-sensitive properties, we investigated the

\footnotetext{
${ }^{1}$ College of Material Science \& Engineering, Donghua University, Shanghai 200051, China

${ }_{2}^{2}$ Material Chemistry Laboratory, Kawamura Institute of Chemical Research, 631 Sakado, Sakura 285-0078, Japan

*To whom correspondence should be addressed (Tel: +81-43-498-2111, Fax: +81-43-498-2182, E-mail: hara@ kicr.or.jp).
} 
a) Gel precursor

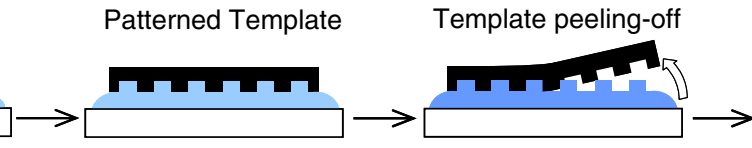

Patterned Gel

Flat substrate

b)

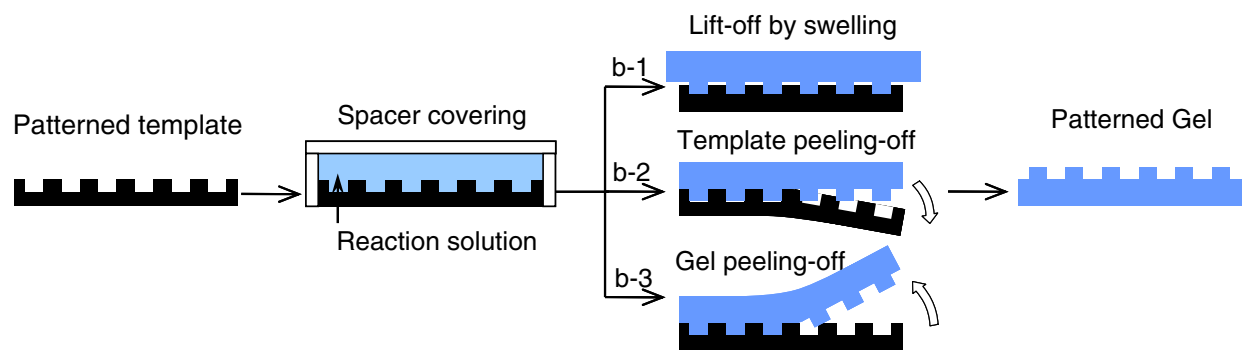

Scheme 1. Process of surface-pattern imprinting by direct replica molding.

preparation of stimulus-sensitive, mechanically tough hydrogel film with a micrometer-scale-patterned surface. We found that surface-patterned NC gel film can be prepared by direct replica molding (direct REM) (Scheme 1(b-3)) without any chemical surface treatment of the template, and that the changes (enlargement and miniaturization) in pattern size can be induced by subsequent swelling, deswelling and drying.

\section{EXPERIMENTAL}

\section{Materials}

$N$-Isopropylacrylamide (NIPA), provided by Kohjin Co., was purified by recrystallization from a toluene $/ n$-hexane mixture and dried in a vacuum at $40{ }^{\circ} \mathrm{C}$. Other reagents were purchased from Wako Pure Chemical Industries, Japan, and used without further purification. As an inorganic clay, synthetic hectorite "Laponite XLG" (Rockwood Ltd.: $\left[\mathrm{Mg}_{5.34} \mathrm{Li}_{0.66} \mathrm{Si}_{8} \mathrm{O}_{20}(\mathrm{OH})_{4}\right] \mathrm{Na}_{0.66}$, layer size $=20-30 \mathrm{~nm} \Phi \times$ $1 \mathrm{~nm}$, cation exchange capacity: 104 mequiv/100 g) was used after washing and freeze-drying. $N, N^{\prime}$-Methylenebis(acrylamide) (BIS) was used as organic crosslinker. $N, N, N^{\prime}, N^{\prime}$ Tetramethylethylenediamine (TEMED) and Potassium peroxodisulfate (KPS) were used as catalyst and initiator respectively. Ultrapure water was used for in situ polymerization supplyed by a Puric-Mx system (Organo Co., Japan). For preparations of hydrogels, nitrogen gas was bubbled through the ultrapure water for more than $3 \mathrm{~h}$ prior to use. Deionized water was distilled for swelling/deswelling experiments.

\section{Preparation of Surface-patterned Gel Film}

The NC gel synthesis procedure was the same as that reported previously. ${ }^{31-35}$ The clay concentration was varied from $0.5 \times 10^{-2}$ to $10 \times 10^{-2} \mathrm{~mol}$ of clay in 1 liter of $\mathrm{H}_{2} \mathrm{O}$. The NIPA concentration was kept at 1 mol per liter of $\mathrm{H}_{2} \mathrm{O}$. In the present paper, the surface-patterning results are shown for $\mathrm{NC}$ gel with $5 \times 10^{-2} \mathrm{~mol}$ of clay, unless otherwise specified.
To prepare the $\mathrm{NC}$ gel, a transparent reaction solution consisting of $19 \mathrm{~g}$ of water, $0.762 \mathrm{~g}$ of inorganic clay (Laponite XLG: $\left.5 \times 10^{-2} \mathrm{~mol}\right)$ and $2.26 \mathrm{~g}(1 \mathrm{M})$ of $N$-isopropyl acrylamide (NIPA), was prepared, followed by the addition of $16 \mu \mathrm{L}$ of catalyst $\left(N, N, N^{\prime}, N^{\prime}\right.$-tetramethylethylenediamine: TEMED) and $1 \mathrm{~g}$ of an aqueous solution of initiator (potassium peroxodisulfate: KPS, $2 \mathrm{wt} \%$ ) at ice-bath temperature. The initial reaction solution was poured onto the patterned template (Scheme 1(b)). In the present study, three kinds of template, one made of stainless steel, one of quartz (hard templates) and one of PDMS (soft template), were used. The stainless steel template had a large stripe pattern with each stripe $350 \mu \mathrm{m}$ wide or a circle dot hole pattern with $350 \mu \mathrm{m}$ diameter. For the quartz templates, three types of pattern designs, one with parallel stripes ( $3 \mu \mathrm{m}$ wide at $15-\mu \mathrm{m}$ intervals), and two with arrays of square dot holes (one with $10-\mu \mathrm{m}$ in length at $10-\mu \mathrm{m}$ intervals and another with $3-\mu \mathrm{m}$ in length at $15-\mu \mathrm{m}$ intervals), were used. All templates were used after washing with ethanol to remove any organic contaminants and drying with a nitrogen stream. Free-radical polymerization was allowed to proceed at $20^{\circ} \mathrm{C}$ for $12 \mathrm{~h}$. Throughout the experiment, oxygen was excluded from the system by vacuum and exchanged with nitrogen. The thickness of the film can be arbitrarily selected using a silicon rubber spacer with different heights ranging from $0.1 \mathrm{~mm}$ to $100 \mathrm{~mm}$ or more. In the present paper, all the prepared films had a thickness of $1 \mathrm{~mm}$ except those shown in Figure $1(2 \mathrm{~mm})$. For comparison, conventional chemicallycrosslinked PNIPA hydrogel (abbreviation: OR gel) film was also prepared using the same procedure as that for $\mathrm{NC}$ gel, except for the use of an organic crosslinker $\left(N, N^{\prime}\right.$-methylenebis(acrylamide): BIS, $0.028 \mathrm{~g}: 1 \mathrm{~mol} \%$ relative to NIPA) instead of clay.

Swelling and de-Swelling of Surface Patterned NC Gel Film

After polymerization at $20^{\circ} \mathrm{C}$, surface-patterned $\mathrm{NC}$ gels were obtained by simply peeling off from the templates, as 
a)

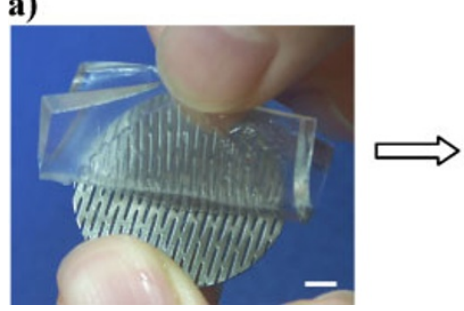

b)

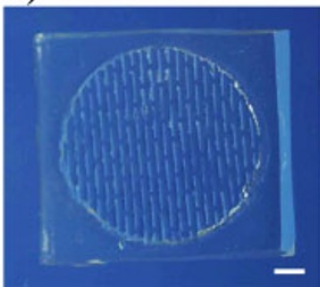

c)

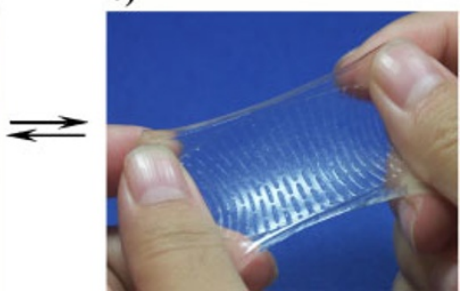

Figure 1. Photos of (a) the process of peeling off of NC gel from a stainless steel template; (b) the resulting surface-patterned NC gel film and (c) the stretching of the patterned NC gel film. Scale: $3 \mathrm{~mm}$.

shown in Scheme 1(b-3). Swelling and de-swelling experiments were performed by immersing surface-patterned gel film in a large excess of distilled water at $20^{\circ} \mathrm{C}$ and at $50^{\circ} \mathrm{C}$, respectively, for $1 \mathrm{~h}$; under these conditions, the gel is very close to the equilibrium state.

\section{Observation of Surface-pattern}

The microstructures of the template and the as-prepared, swollen and deswollen NC gel films were measured using an optical microscope (OM100, Nikon) and a laser scanning confocal microscope (LSCM) (1LM15, LaserTec Corp.). A scanning electron microscope (S-800, Hitachi) was used to observe the microstructure of the corresponding dried $\mathrm{NC}$ gel film.

\section{RESULTS AND DISCUSSION}

Figure 1 shows the manual peeling-off of NC gel film from a template (Figure 1a) and the resulting surface-patterned NC gel film (Figure 1b). It was found that surface-patterned NC gel film can be obtained by a simple procedure, shown in Scheme 1(b-3), without any chemical surface treatment. The pattern of the template was precisely transferred to the surface of the transparent, soft NC gel film. Also, the surface-patterned NC gel films showed high tensile stress $(120 \mathrm{kPa})$ and high elongation at break $(900 \%)$ as reported previously. ${ }^{32}$ So, the resulting surface-patterned NC gel film could be handled without damage and even stretched or bent, as shown in Figure 1c. Since the NC gel could revert almost completely to the original length when the deformation (e.g., stretching) was less than $100 \%$, the change in pattern size caused by the peeling-off was always very small (less than $1 \%$ ).

Figures $2 \mathrm{a}$ and $2 \mathrm{~b}$ show the patterned surface of the quartz template and that of the $\mathrm{NC}$ gel surface, respectively, as observed by LSCM. It was revealed that the micrometer-scale pattern of the template with $3-\mu \mathrm{m}$ stripes (grooves) at $15-\mu \mathrm{m}$ intervals (Figure 2a) was transferred on the surface of the NC gel without any defects (Figure 2b). Because of the outstanding mechanical property (elasticity) of NC gel, the applied stresses and deformation during the removal process did not cause any damage to the pattern, nor did it alter the pattern size.

It has been reported that, in general, hydrophilic gel which is not mechanically tough is difficult to peel off from a hydrophilic template (e.g., silicon wafer) without causing defects
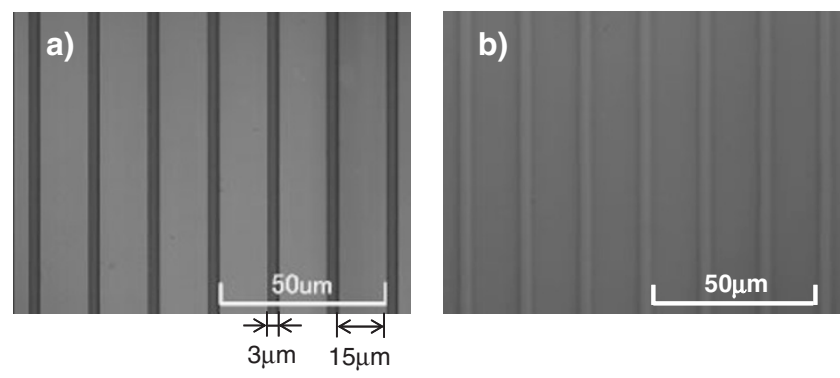

Figure 2. Confocal optical microscopy images of (a) a quartz template pattern with $3-\mu \mathrm{m}$ strips at $15-\mu \mathrm{m}$ intervals and (b) the corresponding surface-patterned NC gel.

because of the strong interaction between the surface of the template and that of the hydrogel. Indeed, the imprinting of a microstructure onto the surface of conventional PNIPA hydrogel (OR gel) was not successful in the present study. Therefore, to achieve the flawless lifting of the hydrogel from the template, hydrophobic modification of the template surface and/or the swelling of the hydrogel have been attempted. ${ }^{27,28}$ However, NC gel could be readily peeled off from the hydrophilic template. This may be partly due to the tough mechanical properties of $\mathrm{NC}$ gel and partly due to the hydrophobic nature of the NC gel surface. The lifting of the NC gel from the template may be facilitated by the abnormally high hydrophobicity (high contact angle for water) of NC gel at the gel-air interface. ${ }^{36}$ In fact, when $\operatorname{poly}(N, N$-dimethylacrylamide)-based NC gel, which possesses a normal hydrophilic surface, is used, its removal from the patterned template is more difficult than the removal of (PNIPA-)NC gel and requires larger stress to achieve detachment.

Confocal optical microscopy images of surface-patterned $\mathrm{NC}$ gel films formed in quartz templates with dot patterns consisting of $10-\mu \mathrm{m}$ squares and $3-\mu \mathrm{m}$ squares at 10 - and 15$\mu \mathrm{m}$ intervals are shown in Figure $3 \mathrm{a}$ and $3 \mathrm{~b}$, respectively. It was found that the same pattern size (error within 1\%) was reproduced on the surface of the NC gels. A three-dimensional (3-D) image and a 2-D profile of the microstructure of the surface-patterned NC gel film with a $10-\mu \mathrm{m}$ square dot array are shown in Figure 3c and 3d, respectively; the 10- $\mu \mathrm{m}$ dot pattern has a height of $1.6 \mu \mathrm{m}$. Since NC gel contains $662 \mathrm{wt} \%$ water against its solid components, it is reasonable to expect the microstructure to shrink due to subsequent drying of the surface-patterned NC gel film. In fact, as observed by SEM 
a)

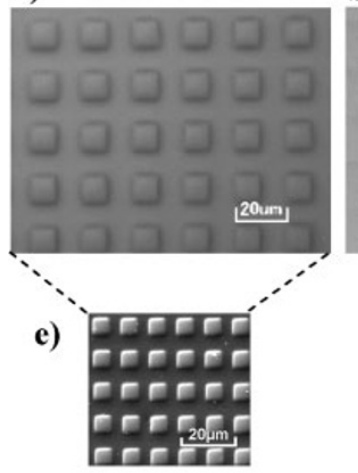

b)

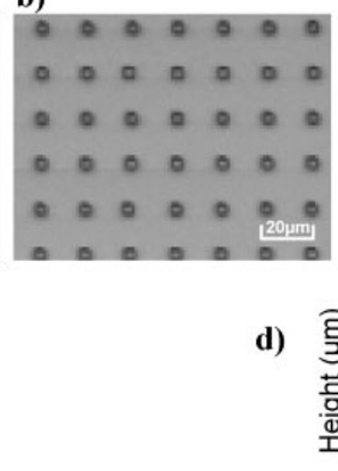

c)
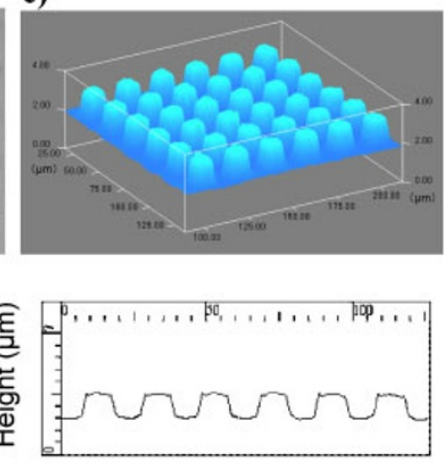

Distance $(\mu \mathrm{m})$

Figure 3. Confocal optical microscopy images of the surface pattern of NC gel films prepared by direct REM using a quartz template with an array of (a) $10-\mu \mathrm{m}$ square dot holes with 10- $\mu \mathrm{m}$ intervals and (b) 3- $\mu \mathrm{m}$ square dot holes with 15- $\mu \mathrm{m}$ intervals. (c) 3-D image ( $\mu \mathrm{m}$ ) and (d) 2-D profile of the surface pattern of the NC gel film shown in (a). (e) SEM image of the miniaturized surface-pattern of the NC gel film shown in (a) which was subsequently dried.

b) de-swollen NC gel

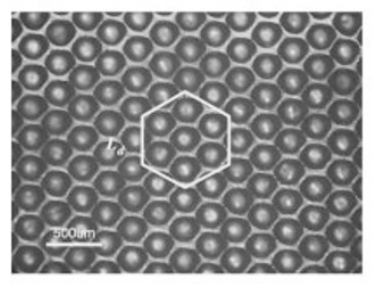

$\sqrt{3}$

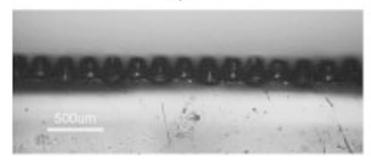

a) as-prepared $\mathrm{NC}$ gel

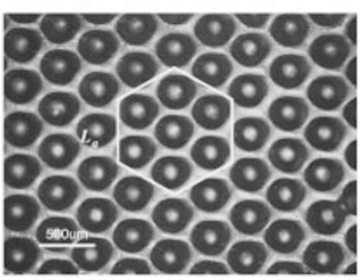

గ

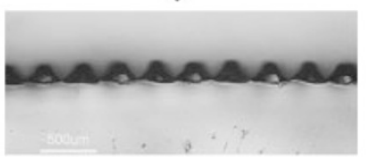

c) swollen NC gel

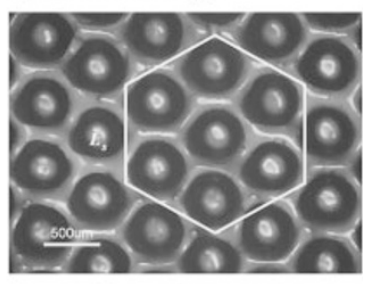

గ

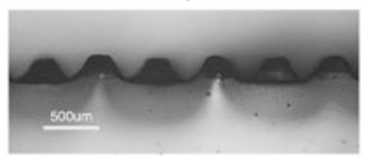

Figure 4. Optical microscopy images of surface-patterned NC gel films in (a) the as-prepared state fabricated using a stainless steel template with an array of dot holes $\left(350 \mu \mathrm{m}\right.$ in diameter), (b) the equilibrium de-swelling state at $50^{\circ} \mathrm{C}$ in water, and (c) the equilibrium swelling state at $20^{\circ} \mathrm{C}$ in water.

(Figure 3e), a miniaturized version of the original surface pattern was realized on the corresponding dried NC gel film. From a comparison of the microstructures of the surfacepatterned NC gel film (Figure 3a) and the corresponding dried one (Figure $3 \mathrm{e}$ ), it was found that the observed pattern size ratio $\left(\mathrm{d}_{\text {gel }} / \mathrm{d}_{\mathrm{dry}}(\mathrm{obs})=1.9\right)$ between the two states was very close to the ratio calculated from the change in water content $\left(\mathrm{d}_{\text {gel }} / \mathrm{d}_{\text {dry }}(\right.$ calc $\left.)=2.1\right)$. Here, $2.65 \mathrm{~g} / \mathrm{cm}^{3}$ and $1.10 \mathrm{~g} / \mathrm{cm}^{3}$ were used as the density of the clay and PNIPA, respectively. This indicates that surface-patterned NC gel film shrinks homogenously in the drying process, yielding a miniaturized surfacepattern in the dried state.

Thus, it was found that micrometer-scale patterns can be precisely transferred to the surface of (PNIPA-) NC gel film by direct REM. Also, surface-patterned NC gel film was similarly prepared using soft PDMS templates. Furthermore, by combining the basic line- or dot-array patterns shown in Figures 2 and 3 , more complicated patterns can be created using the same procedure. In contrast, it was impossible to prepare patterned PNIPA hydrogel films using conventional chemically-crosslinked PNIPA hydrogel (OR gel), because OR gel, which is weak and brittle, always crumbles during the peeling-off process. Also, linear PNIPA solution without clay nanoparticles, which is a very viscous gel-like material, is difficult to peel off from the template due to the solution's stickiness and low mechanical properties.

Since NC gel exhibits a well-defined thermosensitivity, as reported previously, ${ }^{31-35}$ surface-patterned $\mathrm{NC}$ gel films show thermosensitive changes in volume and pattern size. Actually, surface-patterned NC gel film prepared using a stainless steel template with an array of dot holes $(350 \mu \mathrm{m}$ in diameter) could be changed the microstructure upon immersion of the $\mathrm{NC}$ gel film in water at $20^{\circ} \mathrm{C}(<\mathrm{LCST})$ and at $50^{\circ} \mathrm{C}(>$ LCST).

Figure 4(a), 4(b) and 4(c) show the microstructure of the original as-prepared NC gel film with an array of dot holes (average diameter: $347 \mu \mathrm{m}$ ), and those of the gel in the equilibrium de-swollen and swollen state, respectively, as observed through an optical microscope in both surface and cross-section views. Although swollen and de-swollen gels were obtained by immersing the gels in water for $1 \mathrm{~h}$, the time needed for the pattern size change was approximately $10 \mathrm{~min}$. The difference in microstructure size between the original and 
the swollen and de-swollen states are expressed as the ratio of the hexagonal length to that of the original as-prepared state, $L s / L a$ and $L d / L a$, respectively (Figure 4). Here, $L s / L a$ is 1.57 , which is very close to the value $(=1.58)$ calculated from the weight ratio of the swollen gel to the original as-prepared gel $\left(W_{\text {swollen }} / W_{\text {as-prepared }}=3.93\right)$. Similarly, in the de-swollen gel, the ratio of the hexagonal length decreased to 0.75 , which is also very close to the value calculated from the weight change between the two states $(=0.77)$. The consistent changes in surface-pattern size and bulk weight (volume) in the surfacepatterned NC gel film may be ascribed to the uniform expansion and contraction of the PNIPA/clay network consisting of a uniform dispersion of nano-clay particles and flexible PNIPA chains.

Thus, compared with a number of surface-patterned hydrogels reported previously, surface-patterned (PNIPA-based) NC gel film has considerable advantages: fabrication of micrometer-scale surface patterns by simple direct REM without any chemical surface treatment of the template to facilitate the peeling-off process, fine pattern size $(\geq 1 \mu \mathrm{m})$, a broad range of feasible film thicknesses $(0.1 \sim 100 \mathrm{~mm})$, the possibility of using both soft and hard templates, well-defined stimulussensitivity of the surface-patterned hydrogel film, reversible control of the microstructure size by swelling, de-swelling and drying of the original hydrogel, and superior mechanical toughness which can withstand high levels of deformation (stretching, bending, compression, etc.) without damage or change in the microstructure.

Finally, the limitations of NC gel surface patterning should be noted, although they have been not clearly elucidated yet. The pattern size and the concentrations of the ingredients of NC gel may have a lower and/or upper limit. Regarding pattern size, widths of less than $0.5 \mu \mathrm{m}$ and depth/width ratios of more than 0.5 may be difficult to achieve. As for the composition, NC gels with very low and very high clay concentrations, i.e., $<1 \times 10^{-2} \mathrm{~mol}$ and $>8 \times 10^{-2} \mathrm{~mol}$ of clay in 1 liter of $\mathrm{H}_{2} \mathrm{O}$, hardly allow micrometer-scale surface patterning, mainly due to the high viscosity of the initial reaction solution and the very high stickiness and poor mechanical properties (low stress and low modulus) of the resulting $\mathrm{NC}$ gel.

\section{CONCLUSION}

In conclusion, we successfully demonstrated the fabrication of microstructures on the surface of PNIPA-based nanocomposite hydrogel (NC gel) films by direct replica molding. Surface-patterned NC gel films with different thicknesses were prepared using both soft and hard templates and without any chemical surface treatment of the templates to facilitate the peeling-off process. Microstructures in a small size $(\sim 1 \mu \mathrm{m})$ were precisely transferred onto the surfaces of the $\mathrm{NC}$ gel films, and the size could be modified (enlargement and miniaturization) without disturbing the pattern by subsequent swelling, thermosensitive deswelling and drying. The changes in size were consistent with those calculated from the weight changes of the bulk gel film. Thus, NC gel with extraordinary mechanical toughness as well as high transparency and excellent stimulus sensitivity could be formed into surfacepatterned NC gel films by direct replica molding; this may be highly useful for many applications such as selective absorption, controlled drug delivery, rapidly responsive sensors and actuators, effective cell cultivation and cell encapsulation, and microchannels for micro-reactors and micro-analysis.

Received: January 31, 2008

Accepted: May 7, 2008

Published: July 9, 2008

\section{REFERENCES}

1. P. S. Stayton, T. Shimoboji, C. Long, A. Chilkoti, G. Chen, J. M. Harris, and A. S. Hoffman, Nature, 378, 472 (1995).

2. C. M. Hassan, F. J. Doyle III, and N. A. Peppas, Macromolecules, 30, 6166 (1997).

3. A. J. Marshall, J. Blyth, C. A. B. Davidson, and C. R. Lowe, Anal. Chem., 75, 4423 (2003).

4. J. Kim, M. J. Serpe, and L. A. Lyon, J. Am. Chem. Soc., 126, 9512 (2004).

5. O. Steinbock and A. E. Stiegman, Angew. Chem., Int. Ed. Engl., 43, 1505 (2004).

6. K. Haraguchi, T. Takehisa, and M. Ebato, Biomacromolecules, 7, 3267 (2006).

7. H. G. Schild, Prog. Polym. Sci., 17, 163 (1992).

8. R. Yoshida, K. Uchida, Y. Kaneko, K. Sakai, A. Kikuchi, Y. Sakurai, and T. Okano, Nature, 374, 240 (1995).

9. J. H. Holtz and S. A. Asher, Nature, 389, 829 (1997).

10. R. A. Stile, W. R. Burghardt, and K. E. Healy, Macromolecules, 32, 7370 (1999).

11. S. Rimmer, S. Carter, R. Rutkaite, J. W. Haycock, and L. Swanson, Soft Matter, 3, 971 (2007).

12. M. Zourob, J. E. Gough, and R. Ulijin, Adv. Mater., 18, 655 (2006).

13. D.-H. Kim, P. Kim, I. Song, J. M. Cha, S. H. Lee, B. Kim, and K. Y. Suh, Langmuir, 22, 5419 (2006).

14. A. Szilagyi, K. Sumaru, S. Sugiura, T. Takagi, T. Shinbo, M. Zrinyi, and T. Kanamori, Chem. Mater., 19, 2730 (2007).

15. Z. Hu, Y. Chen, C. Wang, Y. Zheng, and Y. Li, Nature, 393, 149 (1998).

16. G. Chen, Y. Imanishi, and Y. Ito, Langmuir, 14, 6610 (1998).

17. D. J. Beebe, J. S. Moore, J. M. Bauer, Q. Yu, R. H. Liu, C. Devadoss, and B.-H. Jo, Nature, 404, 588 (2000).

18. A. Revzin, R. J. Russll, V. K. Yasavalli, W.-G. Koh, C. Deister, D. D. Hile, M. B. Mellott, and M. V. Pishko, Langmuir, 17, 5440 (2001).

19. H. R. Allcock, M. V. B. Phelps, E. W. Barrett, M. V. Pishko, and W.-G. Koh, Chem. Mater., 18, 609 (2006).

20. E. Kim, Y. N. Xia, and G. M. Whitesides, Nature, 376, 581 (1995).

21. Y. N. Xia and G. M. Whitesides, Angew. Chem., Int. Ed. Engl., 37, 550 (1998).

22. K. Y. Suh, J. Seong, A. Khademhosseini, P. E. Laibinis, and R. Langer, Biomaterials, 25, 557 (2004).

23. A. Castellanos, S. J. Dupont, A. J. Heim II, G. Matthews, P. G. Stroot, W. Moreno, and R. G. Toomey, Langmuir, 23, 6391 (2007).

24. F. D. Benedetto, A. Biasco, D. Pisignano, and R. Cingolani, Nanotechnology, 16, S165 (2005).

25. G. T. Franzesi, B. Ni, Y. Ling, and A. Khademhnosseini, J. Am. Chem. Soc., 128, 15064 (2006).

26. M. D. Tang, A. P. Golden, and J. Tien, J. Am. Chem. Soc., 125, 12988 (2003).

27. F. Chiellini, R. Bizzarri, C. K. Ober, D. Schmaljohann, T. Yu, and R. Solaro, Macromol. Rapid Commun., 22, 1284 (2001).

28. T. Yu and C. K. Ober, Biomacromolecules, 4, 1126 (2003). 
29. M. Mayer, J. Yang, I. Gitlin, D. H. Gracias, and G. M. Whitesides, Proteomics, 4, 2366 (2004).

30. M. M. Stevens, M. Mayer, D. G. Anderson, D. B. Weibel, G. M. Whitesides, and R. Langer, Biomaterials, 26, 7636 (2005).

31. K. Haraguchi and T. Takehisa, Adv. Mater., 14, 1120 (2002).

32. K. Haraguchi, T. Takehisa, and S. Fan, Macromolecules, 35, 10162 (2002).
33. K. Haraguchi and H. J. Li, Angew. Chem., Int. Ed., 44, 6500 (2005),

34. K. Haraguchi and H. J. Li, Macromolecules, 39, 1898 (2006).

35. K. Haraguchi, H. J. Li, L. Y. Song, and K. Murata, Macromolecules, 40, 6973 (2007).

36. K. Haraguchi, H. J. Li, and N. Okumura, Macromolecules, 40, 2299 (2007). 